\title{
Aurora kinase A in gastrointestinal cancers: time to target
}

\author{
Ahmed Katsha', Abbes Belkhiri', Laura Goff ${ }^{3}$ and Wael El-Rifai ${ }^{1,2,4^{*}}$
}

\begin{abstract}
Gastrointestinal (Gl) cancers are a major cause of cancer-related deaths. During the last two decades, several studies have shown amplification and overexpression of Aurora kinase A (AURKA) in several GI malignancies. These studies demonstrated that AURKA not only plays a role in regulating cell cycle and mitosis, but also regulates a number of key oncogenic signaling pathways. Although AURKA inhibitors have moved to phase III clinical trials in lymphomas, there has been slower progress in Gl cancers and solid tumors. Ongoing clinical trials testing AURKA inhibitors as a single agent or in combination with conventional chemotherapies are expected to provide important clinical information for targeting AURKA in Gl cancers. It is, therefore, imperative to consider investigations of molecular determinants of response and resistance to this class of inhibitors. This will improve evaluation of the efficacy of these drugs and establish biomarker based strategies for enrollment into clinical trials, which hold the future direction for personalized cancer therapy. In this review, we will discuss the available data on AURKA in GI cancers. We will also summarize the major AURKA inhibitors that have been developed and tested in pre-clinical and clinical settings.
\end{abstract}

Keywords: Aurora kinases, Therapy, AURKA inhibitors, MNL8237, Alisertib, Gastrointestinal, Cancer, Signaling pathways

\section{Introduction}

Mitotic kinases are the main proteins that coordinate accurate mitotic processing [1]. In lower organisms, mitotic kinases include polo-like kinases, NIMA-related kinases, cyclin-dependent kinase 1, WARTS/LATS1-related kinases, and Aurora/Ipl1-related kinases [2]. In humans, the aurora kinase family is considered homologous to Aurora/Ipl1 kinases [2]. This family consists of highly conserved serine-threonine kinases, which play a critical role in regulation of mitotic events like spindle assembly, function of centrosomes and cytoskeleton, and cytokinesis [3,4]. Members of this family are Aurora kinase A (AURKA), Aurora kinase B (AURKB), and Aurora kinase C (AURKC) [3,4]. AURKA is located at chromosome $20 \mathrm{q} 13.2[5,6]$ and was found to play an important role during mitosis. It has been shown that AURKA knockdown suppresses centrosome maturation [7,8]. In mice, AURKA genetic ablation or null mutation leads to mitotic arrest and embryonic death at the blastocyst

\footnotetext{
* Correspondence: wael.el-rifai@vanderbilt.edu

'Department of Surgery, Vanderbilt University Medical Center, 760 PRB, 2220 Pierce Avenue, 37232-6308 Nashville, TN, USA

2Department of Cancer Biology, Vanderbilt University Medical Center, Nashville, TN, USA

Full list of author information is available at the end of the article
}

stage [9-11]. Furthermore, AURKA is critical for bipolar-spindle assembly where it interacts with RanTPX2 pathway [12]. In addition to its role in mitosis, AURKA is recently reported to regulate stem cell selfrenewal, reprogramming, and differentiation $[13,14]$. Overexpression of AURKA has been reported to impair DNA damage repair through inhibition recruitment of RAD51 to double strands breaks [15]. Additionally, AURKA was found to suppress BRCA1 and BRCA2, both play a major role in DNA damage response $[16,17]$. Furthermore, AURKA was also reported to regulate cell migration and adhesion [18] and to mediate an increase in VEGF mRNA expression [19]. AURKA activity is dependent on its phosphorylation at the 288-threonine residue (T288); located on the activation loop of the kinase. AURKA is activated through an autophosphorylation process upon binding with TPX2 $[12,20]$, along with several other kinases. AURKA signaling networks are summarized in Table 1 and Figure 1.

Several studies have shown that AURKA is frequently amplified in several tumors including breast, leukemia, 
Table 1 Selected cancer associated proteins regulated by AURKA

\begin{tabular}{|c|c|c|c|}
\hline Protein & Type of regulation & Functional significance & References \\
\hline \multirow[t]{2}{*}{$\overline{p 53}$} & Phospho-Ser215 & Abrogates transactivation & {$[48,56]$} \\
\hline & Phospho-Ser315 & Abrogates transactivation and enhances proteasomal degradation & \\
\hline NF-kBp65 & Phospho-Ser536 & Promotes inflammation and cell survival & {$[49]$} \\
\hline P73 & Phospho-Ser235 & Induces apoptosis & {$[52-56]$} \\
\hline MDM2 & Phospho-Ser166 & Activates MDM2 and degradation of P53 & [48] \\
\hline GSK3 $\beta$ & Phospho-Ser9 & Inhibits GSK3 $\beta$ and enhances $\beta$-catenin activity & {$[19]$} \\
\hline $\mathrm{IKBa}$ & Phospho-Ser32 & Induces degradation of $\mathrm{IKBa}$ and activation of NF-KB pathway & [49] \\
\hline JAK2 & Increased expression & Activates STAT3 & [55] \\
\hline N-MYC & Increased expression & Stabilizes N-MYC protein & {$[106]$} \\
\hline SRC & Phospho-Tyr416 & Activates SRC and promotes cancer cell invasion & [18] \\
\hline $\mathrm{IKKa} / \beta$ & Phospho-Ser176/180 & Activates NF-kB pathway & {$[107]$} \\
\hline NF-kB2/p100 & Phospho-Ser866/870 & Activates non-canonical NF-kB pathway & {$[108]$} \\
\hline STAT3 & Phospho-Tyr705 & Promotes cell survival & [55] \\
\hline RalA & Phospho-Ser194 & Enhances transformed cell growth & [74] \\
\hline BRCA1/BRCA2 & Decreased expression & Down-regulate cell cycle and DNA damage response & {$[16,17,109]$} \\
\hline
\end{tabular}

bladder, ovarian, gastric, esophageal, liver, colorectal, and pancreatic cancers [21-30]. Notably, AURKA overexpression has been significantly associated with high-grade (grade II-IV) and high-stage (stage IIIB-IV) hepatocellular carcinoma tumors [30]. Furthermore, it was suggested that AURKA is an essential mediator of chemoresistance in colorectal and breast cancers [31,32]. Importantly, several studies indicated that AURKA overexpression directly leads to malignant transformation and tumor formation [25,33,34]. Additionally, AURKA inhibition or knockdown was shown to induce senescence in multiple myeloma [35] and colon cancer cells [36]. Together, these findings strongly suggest the key role of AURKA in tumorigenesis, and thus, underscore AURKA as an attractive target for cancer therapy.

Gastrointestinal (GI) Cancers are a leading cause of cancer-related deaths in the world. In fact, gastric cancer is the second cause of cancer deaths [37], whereas esophageal neoplasm is the eighth most common cancer worldwide and its incidence, especially adenocarcinoma type, is continuously increasing [38]. At the same time, colorectal cancer is the third most common cancer worldwide [39]. In 2013, more than 30,000 new cases of liver cancer were estimated in men and women in the United States [40]. Similarly, more than 45,000 new cases of pancreatic cancer were estimated in the US in 2014 while its survival rate hardly improved in the last three to four decades [40]. The development of new treatment modalities and targeted therapy approaches, based on the molecular features of the tumor, is urgently needed to improve the dismal survival rates of these highly prevalent cancers worldwide. This review will focus on AURKA as a potential molecular therapeutic target in GI cancers. It will also summarize the recent advancement of AURKA inhibitors in pre-clinical studies and clinical trials.

\section{AURKA in gastrointestinal cancers}

Cancers can develop in any anatomical site of the GI system including esophagus, stomach, small intestine, colon, rectum, liver, gall bladder, and pancreas. A cancer in any of these organs represents a unique type where different molecular mechanisms of tumorigenesis can exist. Recent studies have shown that AURKA is frequently overexpressed and/or amplified in several GI cancers [25-28]. In the following sections, we will review and discuss some of the recently reported studies about AURKA functions in GI cancers.

\section{AURKA in upper gastrointestinal cancers}

Upper gastrointestinal (UGI) cancers include tumors of the stomach and esophagus. While esophageal squamous cell carcinoma (ESCC) is the predominant histological type of esophageal cancers in the world, there has been a significant increase in the incidence of gastroesophageal reflux-related esophageal adenocarcinomas (EAC) in the Western world [41]. The majority of gastric cancers are adenocarcinomas; other subtypes such as lymphomas and gastrointestinal stromal tumors are rare and will not be discussed in this review. Amplifications of chromosomal region 20q13, where AURKA is located, have been described in gastric and esophageal cancers [42,43]. Polymorphisms in the AURKA gene have also been reported in UGI cancers $[44,45]$. Several studies have shown frequent overexpression of AURKA at the mRNA and protein levels in ESCC, EAC, and gastric cancers [28,46-49]. 


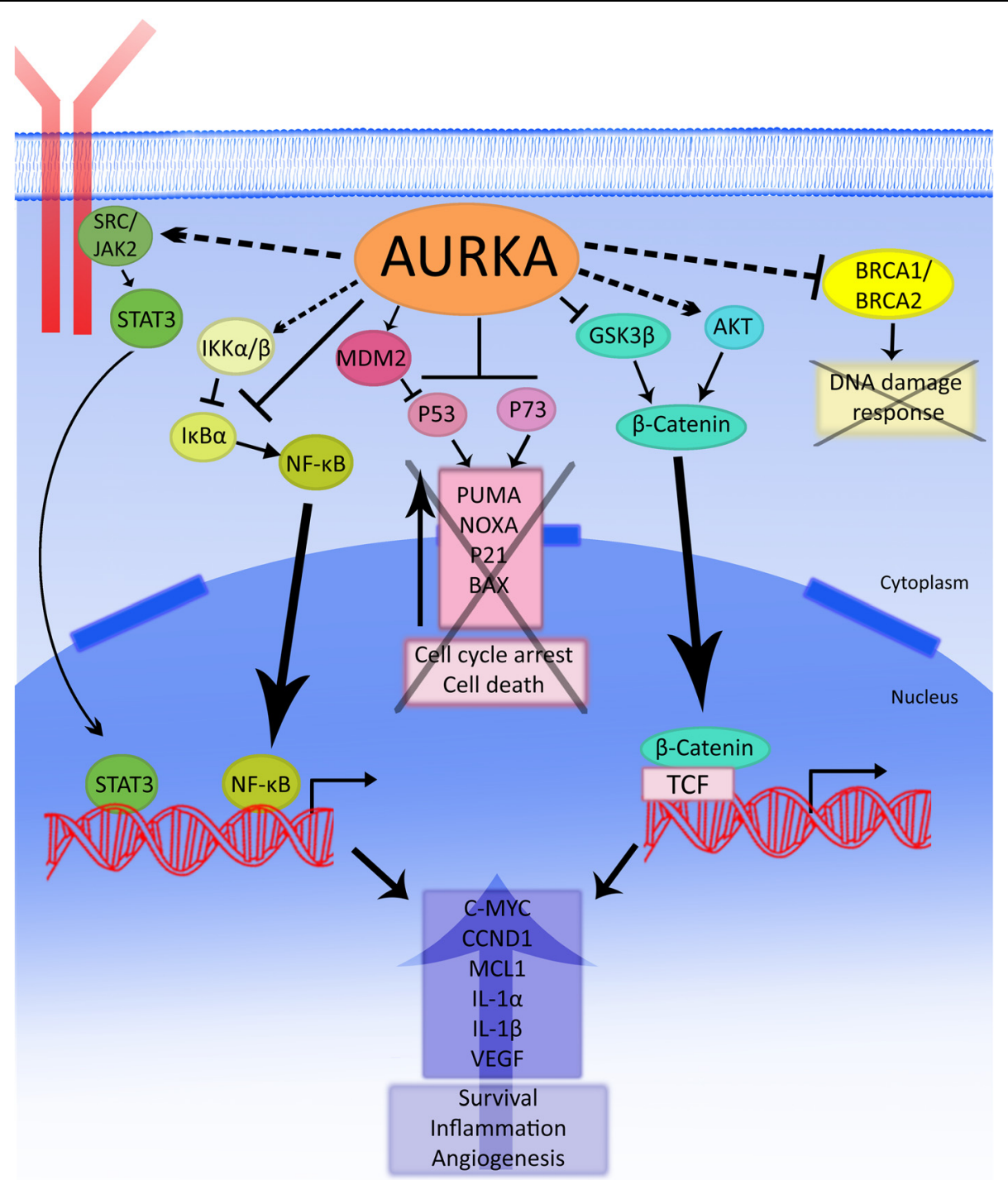

Figure 1 A summary of signaling networks of AURKA in cancer. Solid lines indicate direct regulation, whereas dashed lines indicate indirect regulation.

It has also been suggested that the increased AURKA expression might be used as a prognostic marker of ESCC [50]. In addition, overexpression of AURKA in premalignant and malignant histological stages of reflux-induced Barrett's tumorigenesis has been reported [51]. Similarly, overexpression of AURKA has been observed in early pre-neoplastic stages of gastric cancer in mouse models and human [49]. Taken together, these reports strongly suggest a critical role for AURKA in carcinogenesis.

Molecular studies have shown that AURKA promotes cell survival signaling while it suppresses the proapoptotic machinery in UGI cancer cells $[52,53]$. We and others have reported that AURKA promotes activation of the AKT pro-survival signaling pathway [47,54].
Furthermore, it has been shown that AURKA can regulate and suppress GSK3 $\beta$ kinase activity in gastric cancer cell lines [19]. The AURKA-GSK3 $\beta$ interaction activates $\beta$ catenin/TCF transcription complex, which leads to increased mRNA expression of several oncogenic proteins such as CCND1, c-MYC, c-MYC-binding protein, CLDN1, FGF18, and VEGF [19]. Pro-inflammatory signaling is a significant component in the etiology of tumor development and progression of UGI cancers. In this context, recent reports indicated that AURKA overexpression promotes activation of NF- $\mathrm{kB}$ [49] and STAT3 [55] pathways in these cancers. In these studies, AURKA inhibition abrogates the pro-survival and proinflammatory signaling mediated by NF- $\mathrm{kB}$ and STAT3 
with suppression of key pro-survival target genes such as $B C L-x L, B C L 2$, and MCL1 $[49,55]$. Some of the main features of cancer cells include resistance to proapoptotic stimuli and the ability to overcome genotoxic stress and chemotherapeutic-induced death. We and others have shown that AURKA can suppress p53 pro-apoptotic functions $[48,56]$. A recent study has shown that AURKA regulates the key ubiquitin ligase involved in degradation of p53, HDM2, whereby overexpression of AURKA enhanced HDM2 stabilization and promoted degradation of $\mathrm{p} 53$, thereby abrogating p53 pro-apoptotic function in response to chemotherapy [48]. Not only does AURKA circumvent p53 pathway, but it also provides a mechanism for cancer cells to evade apoptosis by suppressing p73, an important p53 family protein [52,56]. Collectively, these signaling mechanisms mediated by AURKA can promote cancer cell survival and provide a chemoresistance phenotype. These findings also suggest AURKA as part of a signaling hub controlling several key pathways that regulate the hallmarks of the cancer cell network.

Several AURKA inhibitors have been developed to overcome AURKA-mediated pro-survival and antiapoptotic activities in cancer cells. Inhibition of AURKA using investigational MLN8054 or MLN8237 led to reversal of anti-apoptotic signaling cascades with activation of pro-apoptotic p73 in p53 deficient cells with reexpression of several pro-apoptotic proteins such as PUMA, NOXA, and p21 [52]. AURKA inhibition by small molecule MLN8237 as a single agent or in combination with Cisplatin or Docetaxel significantly enhanced cell death in esophageal adenocarcinoma xenograft mouse model [53,57]. These findings strongly indicate that AURKA could be an important therapeutic target in upper GI cancers.

\section{AURKA in colorectal cancer}

Colorectal cancer (CRC) was one of the first cancers found to have AURKA amplification. A 1998 study showed that overexpression of AURKA resulted in centrosome amplification, chromosomal instability and transformation in mammalian cells, including colon cancer cells [34]. Genetic variations in the AURKA gene were also detected and associated with aneuploidy in human colon tumors and low penetrance CRC susceptibility factor [58,59]. AURKA overexpression was associated with the down-regulation of checkpoint with forkhead and ring finger domains (CHFR) in colorectal cancers with high microsatellite instability [60]. Importantly, recent reports indicated that AURKA is critical for tumorigenicity and chemoresistance in CRC stem cells [31], and suggested AURKA as a predictive marker for recurrence in stage III in colon tumors lacking microsatellite instability [61]. These studies, as well as other studies, focused mainly on the fact that overexpression of AURKA leads to chromosomal instability. In normal cells, expression of AURKA protein is predominantly confined to the nucleus and subject to spatio-temporal regulation during mitotic progression. In contrast, expression of AURKA protein is frequently observed in the cytoplasm, in addition to the nucleus, in cancer cells, suggesting an extended role of AURKA beyond regulation of mitosis [62]. This finding provides additional evidence supporting the wealth of signaling data for AURKA reported in upper GI cancers (summarized in the section above). For instance, Tseng and colleagues found that AURKA enhances formation and aggregation of mutant Ras (RasV12) through regulation of RAS/MEK/ERK pathways and AKT phosphorylation in colon cancer [63]. Aurora kinase pharmacological inhibition sensitized colon cancer cells to Tumor necrosis factor (TNF) and TNF-related apoptosis-inducing ligand (TRAIL) [64]. In addition, AURKA inhibition, using specific or pan Aurora kinase inhibitors, sensitized colon cancer cells to chemotherapy [65], TNF or TRAIL induced apoptosis [64] as well as mitotic failure and cell death when inhibited along with SRC [66]. Other reports showed that pharmacological inhibition of AURKA with MLN8054 in colon cancer HCT-116 xenografts induces senescence in vivo [36]. These findings suggest that more studies are needed to better characterize the role of AURKA in colon cancer signaling and therapeutics.

\section{AURKA in pancreatic cancer}

Overexpression of AURKA was reported in pancreatic cancer [26], in the early stages of abnormalities in pancreatic ducts and ductal dysplasia in transgenic mouse model for pancreatic cancer [67], and linked to chromosome instability and centrosome abnormality [68]. As observed in other GI cancers, suppression of AURKA using RNA interference [69] or small molecule inhibitor SNS-314 [70] suppresses tumor growth and enhances chemosensitivity. Similar results were obtained with specific AURKA inhibitor CYC3 in combination with Paclitaxel [71]. In addition, AURKA plays a role in protecting human pancreatic ductal adenocarcinoma cells from autophagic cell death induced by metabolic stress [72]. Interestingly, while it was shown in colorectal cancer that AURKA regulates MEK/ERK pathway [63], it was reported that AURKA is a downstream target of this pathway in pancreatic cancer [73], raising the possibility of an existing positive feedback loop between AURKA and MEK/ERK pathway. This signaling loop further underscores the potential therapeutic benefit of targeting AURKA in pancreatic cancer. Additionally, AURKA can phosphorylate RalA at Ser194 and regulate its subcellular localization, which is essential for Ras- 
mediated cellular transformation [74]. However, further studies by the same group failed to show correlation of RalA (S194) phosphorylation with responsiveness to MLN8237, indicating that the principal target of AURKA may not be RalA [75].

\section{AURKA in liver cancer}

In human hepatocellular carcinoma (HCC), AURKA was found to be overexpressed at mRNA level in $61 \%$ of HCC samples [30], whereas gene amplification was recorded in only 3 out of 224 HCC samples [30]. Notably, high expression levels of AURKA were associated with high-grade, high-stage tumors and poor outcome. Microarray studies suggested that AURKA phosphorylates and stabilizes hepatoma up-regulated protein (HURP) [76], a cell cycle regulated gene that is overexpressed in human hepatocellular carcinoma (HCC) [77]. On the other hand, it was reported that hypoxia and hypoxiainducible factor-1 (HIF-1) are important in the tumor microenvironment and could regulate AURKA at the transcriptional level $[78,79]$. Other studies showed that AURKA, can in turn, regulate HIF- $1 \alpha$ through activation of AKT and p38-MAPK signaling pathways leading to malignant phenotypes of HCC [80], indicating a positive feedback loop sustaining continuous activation of AURKA. Several preclinical studies investigated the effects of AURKA inhibition in HCC. For example, a report showed that the pan Aurora inhibitor PHA-739358 inhibits HCC tumor growth in a tumor xenograft mouse model [81]. Further, AURKA inhibition with small molecule R1498 was found to target mitosis pathway and suppress angiogenesis [82]. These studies emphasize the multifaceted roles of AURKA in HCC and imply AURKA as a feasible druggable target in HCC.

\section{Clinical development of aurora kinase A inhibitors}

The aforementioned preclinical studies shed light on the roles and contributions of AURKA in tumorigenesis, which serve as premise for the development of several pan Aurora kinase inhibitors (AKIs) and selective inhibitors of AURKA. In the following section, we will focus on the present investigational AURKA inhibitors in completed, ongoing, or planned clinical trials.

\section{MLN8237 (alisertib)}

MLN8237 (alisertib) is a second generation derivative of the initial small molecule MLN8054, developed by Millennium Pharmaceuticals, Inc. Both generations work as highly specific ATP-competitive AURKA inhibitors with an IC50 of $2 \mathrm{nM}$ in chronic myeloid leukemia (CML) for MLN8237 [83,84]. The MLN8237 IC50 values vary in other cancers, ranging from $6.7 \mathrm{nM}$ in HeLa cells to $469 \mathrm{nM}$ in DLD-1 colon cancer cell line [85]. MLN8237 can target AURKB at higher doses (396.5 nM) [35,85].
The first generation MLN8054 was terminated in phase 1 clinical trials due to off-target toxicities [86]. Both drugs are ATP competitive, where they bind and inhibit AURKA phosphorylation at T288. In murine xenograft models, MLN8237 was well tolerated with doses ranging from 15 to $40 \mathrm{mg} / \mathrm{kg} / \mathrm{d}$. MLN8237 was also found to be well absorbed and steady state plasma levels were reached within 7 days of twice daily dosing in phase 1 clinical trials [87]. Investigations of MLN8237 in preclinical studies have shown that it induces abnormal G2/M cell cycle arrest, abnormalities in mitotic spindles, and apoptosis in upper GI cancers [48,49,53]. In addition, MLN8237 down-regulates several oncogenic pathways such as NF- $\mathrm{kB}[49,88]$, AKT [19], and STAT3 [55] in cancer cells. On the other hand, several reports demonstrated that treatment with MLN8237 could activate p53 $[47,48,89]$ and p73 $[52,56]$ apoptotic pathways. The effects of MLN8237 were tested in several non-GI cancers. It has been shown that MLN8237 sensitized PC3 and DU145 prostate cancer cells to radiation, increased the DNA double-strand breaks, induced G2/M cell cycle arrest and polyploidy [90]. Treatment of OVCAR-5 and SKOV3ip2 ovarian cancer cells with MLN8237 resulted in inhibition of cytoskeletal regulatory SRC (assessed by decreased phosphorylation at Tyr-416) and decreased migration and adhesion [18]. Moreover, MLN8237 has been tested on many pediatric cancer cell lines like Ewing sarcoma, neuroblastoma, glioblastoma, rhabdomyosarcoma, ALL, and AML where it inhibited cell growth and delayed tumor growth [91]. In vitro and in vivo tumor xenografts mouse models showed that MLN8237 enhances anti-tumor activity of chemotherapeutics. For instance, dual treatment of MLN8237 with Docetaxel enhanced apoptosis and anti-tumor activity in lymphoma [92] and UGI adenocarcinomas cell models [53]. Similarly, a combination of Cisplatin with MLN8237 had robust anti-tumor activity in tumor xenograft models of esophageal adenocarcinomas [57]. In Bcell Non-Hodgkin Lymphoma, MLN8237 in combination with Vincristine and Rituximab showed robust cell death in vitro and had curative effects in vivo using mantle cell lymphoma (MCL) mouse xenograft model [93]. Collectively, the present data indicate that MLN8237 is an effective drug with high specificity and limited off target activity that works in various models. Notably, there are 49 clinical trials with MLN8237 in leukemia, breast, lung, colorectal, ovarian, and pancreatic cancers (clinicaltrials.gov; March 2015). Phase 1 clinical studies of the drug as a single agent demonstrate preliminary activity where $37 \%$ of patients achieved a best response of stable disease for more than 6 months in some solid tumors (colorectal cancer, chondrosarcoma, leiomyosarcoma, and liposarcoma) with modest toxicity such as nausea, stomatitis, and alopecia among other adverse effects [87]. The recommended 
phase 2 dose of MLN8237 is $50 \mathrm{mg}$ orally given twice daily on days 1-7 of a 21-day cycle for various solid tumors and hematologic malignancies [94]. Phase 2 studies of MLN8237 as a single agent are ongoing in multiple tumor types including lung, breast, ovarian, UGI, and pancreatic cancers (Table 2). In some of the clinical trials, MLN8237 has been tested in combination with other drugs such as Cetuximab and Docetaxel among others (Table 2). A recent phase 2 clinical trial of MLN8237 as a single agent in advanced solid tumors revealed that $9 \%$ of patients (4 out of 47 patients) with gastro-esophageal adenocarcinoma partially responded to the treatment [95]. A newly registered phase 1 clinical trial (NCT02319018) is ongoing to investigate the side effects of MLN8237 in combination with other chemotherapeutic drugs (fluorouracil, leucovorin calcium, oxaliplatin). This clinical trial will be the first to evaluate MLN8237 exclusively in gastrointestinal tumors (clinicaltrials.gov, Table 2).

\section{ENMD-2076}

ENMD-2076 was originally developed by EntreMed, Inc. as a selective AURKA inhibitor with an IC50 of 14 $\mathrm{nM}$ in biochemical assays [96]. However, ENMD-2076 was found to inhibit several other oncogenic kinases at different IC50 including FLT3 (1.86 nM), SRC (20.2 $\mathrm{nM})$, VEGFR3 (15.9 nM), VEGFR2 (58.2 nM), PDGFR $\alpha$ (56.4 $\mathrm{nM})$, and FGFR1 (92.7 nM) [96]. This drug was shown to induce G2/M cell cycle arrest and apoptosis, consistent with AURKA inhibition responses. ENMD2076 was found to be selectively toxic to multiple myeloma (MM) cells but minimally toxic to hematopoietic progenitor cells [97]. Interestingly, exposure as short as 6 hours induces apoptosis and reduces AURKA autophosphorylation at T288; however, this was achieved at doses that also inhibited AURKB and several other kinases [97]. In vitro studies in multiple myeloma showed that treatment with ENMD-2076 inhibited AKT phosphorylation, and thus, altered the activity of some of its targets, including GSK3 $\beta$, survivin, and XIAP [97]. Additionally, ENMD-2076 was shown to down-regulate phosphorylation of STAT3 at Ser-727 [97]. In mouse xenograft models of multiple myeloma, ENMD-2076 showed good tolerability at doses up to $200 \mathrm{mg} / \mathrm{kg}$, and immunohistochemistry analysis revealed reduced Ki67 and increased cleaved caspase-3 expression levels [97]. In a patient-derived xenograft model of CRC, ENMD2076 inhibited tumor growth, which led to regression; this was associated with significant reduction in 18FDG uptake at day 3 and 21 of treatment [98]. In breast cancer, ENMD-2076 was most effective against triplenegative tumors with increased mutant p53 expression [99]. In addition, cells lacking estrogen receptor expression and/or HER2 overexpression were the most sensitive cells to the drug [99]. A completed phase 1 clinical trial indicated that this drug is tolerable at $160 \mathrm{mg} / \mathrm{m}^{2}$ daily administered orally in patients with advanced solid tumors [100]. ENMD-2076 is currently being evaluated in phase 1 and 2 clinical trials, specifically in breast and ovarian cancers (clinicaltrials.gov, Table 2).

\section{MK-5108}

MK-5018 (VX689) is a specific AURKA inhibitor (IC50: $0.046 \mathrm{nM}$ ) developed by Vertex Pharmaceuticals [101]. MK-5018 also inhibits AURKB and AURKC, although at higher doses (200 folds higher than AURKA) with various IC50 values among different cancer cell lines [101]. Tumor xenografts treated with MK-5018 showed significant tumor inhibition at doses of 15 or $30 \mathrm{mg} / \mathrm{kg}$ twice daily for 12 days, indicating the high tolerability of the drug [101]. In addition to a typical AURKA inhibition response (polyploidy and cell cycle arrest), inhibition of NF- $\kappa B$ activity and reduction of cytokine production were observed after treatment of ovarian cancer stem cells with MK-5108 [102]. Further, MK-5108 was investigated in combination with other therapeutic agents. For instance, MK-5108, in combination with histone deacetylase inhibitor, vorinostat, induced lymphoma cell death along with reduction of c-Myc, hTERT, and micro RNA levels in vitro [103]. In a colon cancer xenograft mouse model, MK-5108 was tested in combination with Docetaxel and showed additive anti-tumor activity as compared to animals that received Docetaxel alone [101]. A completed phase 1 clinical trial of MK-5108 as a single agent or in combination with Docetaxel to test its safety, side effects, and tolerability showed moderate toxicities with some clinical activity as a single agent in patients with advanced and/or refractory solid tumors [104] (clinicaltrials.gov, Table 2).

\section{Conclusions}

Since their discovery, aurora kinases have been under constant investigation to uncover their roles in carcinogenesis. Several studies have indicated that AURKA is overexpressed and/or amplified in different malignances relative to normal cells. A large body of literature has shown that overexpression of AURKA mediates several pro-tumorigenic functions in addition to mitosis; thereby suggesting AURKA as a potential therapeutic target.

Several studies [19,48,49,52,55] identified multiple downstream targets of AURKA involved in critical cellular functions. These studies demonstrated that AURKA not only plays a role in regulating cell cycle and mitosis, but also interacts with a number of key oncogenic signaling networks, suggesting that AURKA is located at a signaling hub in cancer cells. Notably, many of these molecular regulations were established in GI cancers. These findings could be applicable to other types of 
Table 2 Selected clinical trials of Aurora kinases inhibitors

Inhibitor Chemical structure

NCT number, phase, status

Cancer type Ref.

NCT01898078, Phase 1, Ongoing

Advanced Solid Tumors; Lymphoma

NCT01045421, Phase 1/Phase 2,

Nonhematological Malignancies

Completed

NCT01091428 Phase 1/Phase 2,

Ongoing (Agent in combination with Paclitaxel)

Ovarian Carcinoma; Fallopian Tube

Cancer; Peritoneal Cancer; Breast

Carcinoma

NCT01471964, Phase 1/Phase 2, Ongoing (Agent in combination with Erlotinib)

Metastatic or Recurrent Non-Small

Cell Lung Cancer

NCT02114229, Phase 2, Ongoing

Malignant Rhabdoid Tumor; Atypical Teratoid Rhabdoid Tumor

NCT00962091, Phase 1, Completed Advanced Solid Tumors

NCT01512758, Phase 1, Completed

NCT02109328, Phase 2, Ongoing

Adult East Asian Patients With Advanced Solid Tumors or Lymphomas

Bladder Cancer; Transitional Cell Carcinoma

NCT01812005, Phase 2, Ongoing (Agent in combination with Rituximab)

Relapsed or Refractory B-Cell NonHodgkin Lymphoma

NCT01397825, Phase 1/Phase 2. Relapsed or Refractory Aggressive BOngoing (Agent in combination with Rituximab and Vincristine)

NCT00500903, Phase 1, Completed

NCT00697346, Phase 1, Completed

\section{Advanced Solid Tumors}

Advanced Hematological Malignancies

NCT01799278, Phase 2, Ongoing

Metastatic Castrate Resistant and Neuroendocrine Prostate Cancer

NCT00807495, Phase 2, Completed Aggressive Non-Hodgkin's Lymphoma

NCT01567709, Phase 1, Ongoing (Agent in combination with Vorinostat)

Relapsed or Recurrent Hodgkin Lymphoma, B-Cell Non-Hodgkin Lymphoma, or Peripheral T-Cell Lymphoma

NCT00651664, Phase 1, Completed. Advanced Solid Tumors; Lymphomas

NCT01695941, Phase 1, Ongoing (Agent in combination with Bortezomib and Rituximab)

Relapsed or Refractory Mantle Cell Lymphoma or B-Cell Low Grade Non-Hodgkin Lymphoma

NCT01154816, Phase 2, Completed Recurrent or Refractory Solid Tumors or Leukemia

NCT02038647, Phase 2, Ongoing (Agent Small Cell Lung Cancer in combination with Placebo and Paclitaxel)

NCT01779843, Phase 1, Ongoing (Agent Acute Myeloid Leukemia in combination with Cytarabine and Idarubicin)

NCT00830518, Phase 2, Completed

Acute Myelogenous Leukemia and High-Grade Myelodysplastic Syndrome

NCT01677559, Phase 1, Ongoing (Agent Advanced Solid Malignancies in combination with Nab-Paclitaxel)

NCT01924260, Phase 1, Ongoing (Agent Solid Tumors or Pancreatic Cancer in combination with Gemcitabine) 
Table 2 Selected clinical trials of Aurora kinases inhibitors (Continued)

NCT01897012, Phase 1, Ongoing (Agent Relapsed or Refractory B-Cell or

in combination with Romidepsin) T-Cell Lymphomas

NCT01034553, Phase 1/Phase 2 Relapsed or Refractory Multiple Ongoing (Agent in combination with Bortezomib)

NCT01482962, Phase 3, Ongoing (Agent in combination with Pralatrexate, Gemcitabine and Romidepsin)

NCT01848067, Phase 1/Phase 2,

Ongoing (Agent in combination with

Abiraterone acetate and Prednisone)

NCT01466881, Phase 2, Ongoing Myeloma

Relapsed/Refractory Peripheral T-Cell Lymphoma

Hormone-Resistant Prostate Cancer

Relapsed or Refractory Peripheral T-Cell Non-Hodgkin Lymphoma

NCT01923337, Phase 1, Ongoing (Agent in combination with Irinotecan Aance hydrochloride)

NCT01714947, Phase 1, Completed Advanced Solid Tumors; Lymphoma

NCT01094288, Phase 1, Ongoing (Agent in combination with Docetaxel)

Advanced Solid Tumors Including Castration-Resistant Prostate Cancer

NCT01639911, Phase 1, Ongoing (Agent Solid Tumors

in combination with Pazopanib)

NCT00853307, Phase 2, Completed

Ovarian, Fallopian Tube, or Peritoneal Carcinoma

NCT01540682, Phase 1, Completed Head and Neck Cancer

(Agent in combination with Cetuximab and radiotherapy)

NCT01316692, Phase 2, Ongoing Unresectable Stage III-IV Melanoma

NCT02187991, Phase 2, Ongoing (Agent in combination with Paclitaxel)

Metastatic or Locally Recurrent Breas

NCT02259010, Phase 1, Ongoing (Agent Advanced Solid Tumors or Relapsed/ in combination with Itraconazole) Refractory Lymphoma

NCT02367352, Phase 1, Ongoing (Agent in combination with Paclitaxel)

East Asian Patients With Advanced Solid Tumors

NCT02319018, Phase 1, Ongoing (Agent Gastrointestinal Tumors

in combination with Leucovorin

Calcium, Fluorouracil, and

Oxaliplatin)

NCT02219789, Phase 1, Ongoing (Agent Hormone Receptor Positive, in combination with Fulvestrant) Metastatic or Advanced Breast Cancer

NCT02186509, Phase 1, Ongoing (Agent Recurrent High-Grade Gliomas with radiation)

NCT01848067, Phase 1\&2, Ongoing Hormone-Resistant Prostate Cancer (Agent with Abiraterone acetate and Prednisone)

NCT01779843, Phase 1, Ongoing (Agent Acute Myeloid Leukemia with Cytarabine and Idarubicin) 
Table 2 Selected clinical trials of Aurora kinases inhibitors (Continued)

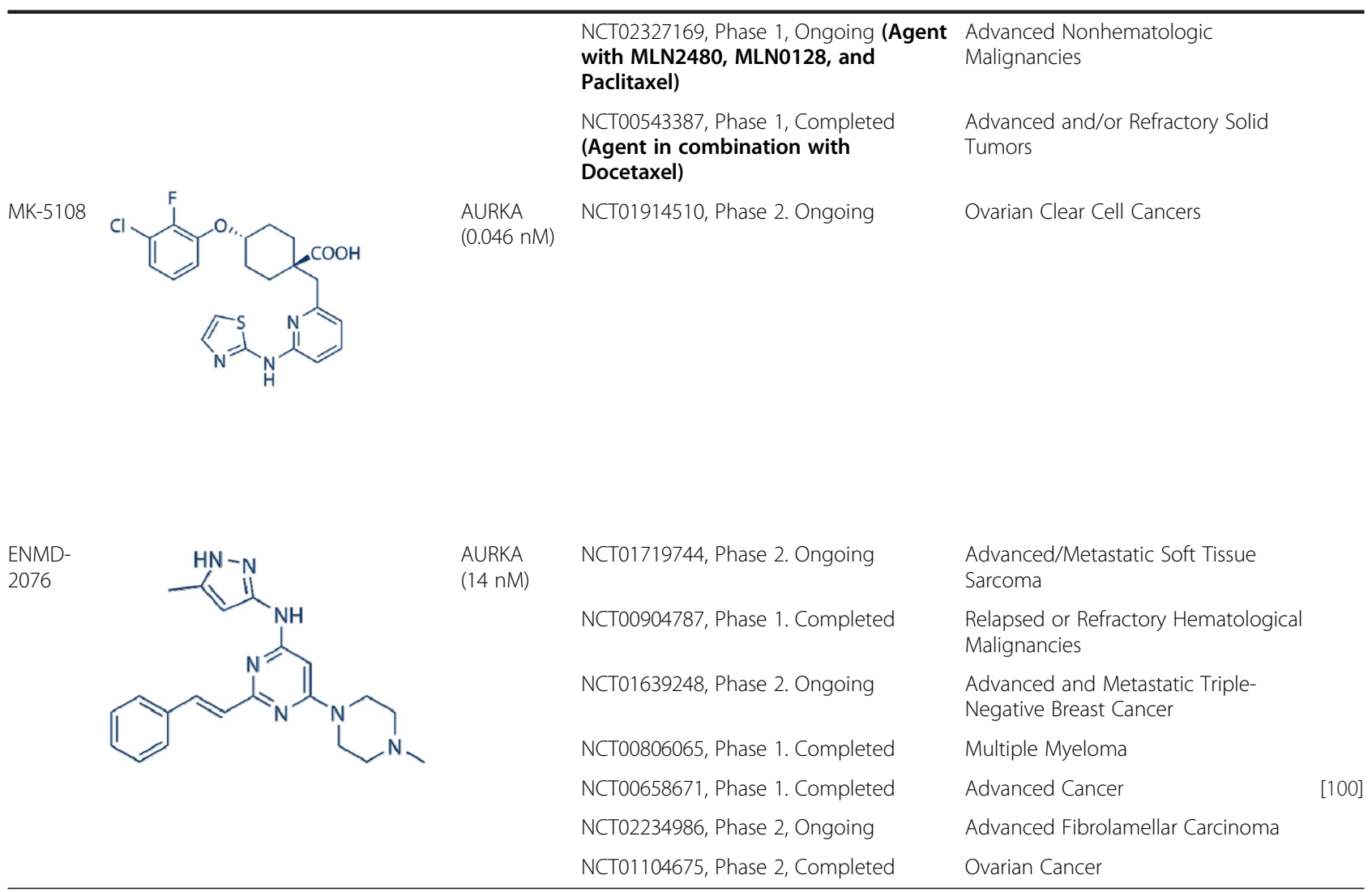

tumors. However, rigorous investigations are required before a conclusion can be drawn. AURKA activates multiple oncogenic signaling pathways while suppressing critical tumor suppressor functions of p53 [48,89] and p73 [52,56] in cancer cells. AURKA regulates several fundamental properties of carcinogenesis such as cell survival, growth, invasion, inflammation, and angiogenesis. Furthermore, AURKA was shown to regulate pluripotency of embryonic stem cells $[13,14]$, which may suggest that AURKA plays a role in regulating stemness features of cancer cells.

Although our understanding of the role of aurora kinases has improved significantly over the past few years, and several pan or specific Aurora inhibitors were developed with variable specificities, only a few have moved forward in clinical trials. One of the most widely studied inhibitors is MLN8237 (alisertib), which has been investigated in preclinical studies using in vitro and tumor xenograft mouse models with promising anti-tumor effects, alone and in combination with Cisplatin [57] or Docetaxel [53]. MLN8237 as single agent or in combination with chemotherapeutics has been evaluated in clinical trials (Table 2). In spite of the extensive preclinical studies on AURKA, few clinical trials were undertaken to evaluate the potentiality of AURKA as a target in GI cancers. The most advanced clinical trial (phase 3 ) is testing MLN8237 in hematologic malignancies (clinical trial\# NCT01482962). In solid tumors, phase 2 clinical trials are currently testing MLN8237 alone or in combination with other chemotherapeutics or biologics (Paclitaxel, Erlotinib, Rituximab, Vincristine among others, Table 2). ENMD-2076 is another specific AURKA inhibitor, currently in phase 2 for ovarian and breast cancers (NCT01914510, NCT01639248, NCT01104675) that has not been tested in GI cancers. Given the experience from other targeted therapy approaches, it is unlikely that AURKA inhibition alone would be sufficient to achieve the desired therapeutic response in solid tumors. Clinical trials utilizing combinations with other anti-cancer drugs may have higher probabilities of success in cancer cell killing by circumventing the expected resistance due to tumor heterogeneity. However, these approaches should be carefully developed as they could be hindered by increased toxicities.

It is also a critical priority to investigate and identify molecular biomarkers of response and resistance to AURKA inhibitors. Biomarker based strategies for patient enrollment into clinical trials hold the future 
direction for personalized cancer therapy. Based on the available literature, several molecules could be potential biomarkers of response or resistance to AURKA inhibition. These molecules include targets of AURKA such as P53, P73, AKT, BRCA 1\&2, STAT3, NF-KB, MDM2, SRC, and MYC $[16,17,48,49,52,55,56]$. For instance, a study aimed to predict biomarkers of response to the aurora kinase inhibitor, PF-03814735, found that the status of the $M Y C$ gene family and retinoblastoma pathway members significantly correlated with the efficacy of the inhibitor in small cell lung cancer and colon cancer cell lines [105]. Identification of biomarkers that can reliably predict benefits from AURKA inhibitors should be developed as an integral part of clinical trials in our pursuit for personalized medicine.

In summary, while there is relatively strong biology and several preclinical studies supporting AURKA as an attractive therapeutic target in GI cancers along with the availability of multiple inhibitors, larger and carefully designed clinical trials will be needed to clearly establish the role of AURKA inhibitors in the treatment of GI cancers.

\begin{abstract}
Abbreviations
Gl: Gastrointestinal; AURKA: Aurora kinase A; AURKB: Aurora kinase B; AURKC: Aurora kinase C; TPX2: Targeting protein for Xklp2; BRCA1/2: Breast cancer susceptibility gene; VEGF: Vascular endothelial growth factor; UGI: Upper gastrointestinal; ESCC: Esophageal squamous cell carcinoma; EAC: Esophageal adenocarcinomas; CCND1: Cyclin D1; FGF: Fibroblast growth factor; STAT3: Signal transducer and activator of transcription 3; BCL-xL: B-cell lymphoma-extra large; BCl2: B-cell lymphoma 2; MCL1: Myeloid Cell Leukemia 1; HDM2: Human double minute 2; PUMA: p53 upregulated modulator of apoptosis; CRC: Colorectal cancer; CHFR: Checkpoint with forkhead and ring finger domains; MAPK: Mitogen-activated protein kinases; ERK: Extracellular signal-regulated kinases; HURP: Hepatoma up-regulated protein; HCC: Human hepatocellular carcinoma; HIF-1: Hypoxia-inducible factor-1; CML: Chronic myeloid leukemia; ALL: Acute lymphoblastic leukemia; AML: Acute myeloid leukemia; MCL: Mantle cell lymphoma; FLT3: FMS-like tyrosine kinase 3; PDGFRa: Alpha-type platelet-derived growth factor receptor; MM: Multiple myeloma; FDG: Fluorodeoxyglucose; HER2: Human epidermal growth factor receptor; hTERT: Human telomerase reverse transcriptase.
\end{abstract}

\section{Competing interests}

The authors declare that they have no competing interests.

\section{Authors' contributions}

AK: Prepared the scientific material, write the main text, generated tables and made figures; AB: revised the scientific contents and edited the text; LG: revised the scientific contents; WE: developed the structure of the review, revised the scientific material, and edited the contents. All authors read and approved the final manuscript.

\section{Acknowledgements}

This review was supported by R01CA131225 from the National Institutes of Health. The contents of this work are solely the responsibility of the authors and do not necessarily represent the official views of the National Cancer Institute or Vanderbilt University.

\section{Author details}

'Department of Surgery, Vanderbilt University Medical Center, 760 PRB, 2220 Pierce Avenue, 37232-6308 Nashville, TN, USA. ${ }^{2}$ Department of Cancer Biology, Vanderbilt University Medical Center, Nashville, TN, USA. ${ }^{3}$ Department of Hematology, Department of Oncology, Vanderbilt University Medical Center, Nashville, TN, USA. ${ }^{4}$ Department of Veterans Affairs, Tennessee Valley Healthcare System, Nashville, TN, USA.
Received: 14 November 2014 Accepted: 28 April 2015

Published online: 20 May 2015

\section{References}

1. Nigg EA. Mitotic kinases as regulators of cell division and its checkpoints. Nat Rev Mol Cell Biol. 2001;2:21-32.

2. Brown JR, Koretke KK, Birkeland ML, Sanseau P, Patrick DR. Evolutionary relationships of Aurora kinases: Implications for model organism studies and the development of anti-cancer drugs. BMC Evol Biol. 2004;4:39-49.

3. Kollareddy M, Dzubak P, Zheleva D, Hajduch M. Aurora Kinases: Structure, Functions and Their Association with Cancer. Biomed Pap Olomouc. 2008;152:27-33.

4. Carmena M, Earnshaw WC. The cellular geography of aurora kinases. Nat Rev Mol Cell Biol. 2003;4:842-54.

5. Sen S, Zhou HY, White RA. A putative serine/threonine kinase encoding gene BTAK on chromosome 20q13 is amplified and overexpressed in human breast cancer cell lines. Oncogene. 1997;14:2195-200.

6. Tanner MM, Grenman S, Koul A, Johannsson O, Meltzer P, Pejovic T, et al. Frequent amplification of chromosomal region 20q12-q13 in ovarian cancer. Clin Cancer Res. 2000;6:1833-9.

7. Hannak E, Kirkham M, Hyman AA, Oegema K. Aurora-A kinase is required for centrosome maturation in Caenorhabditis elegans. J Cell Biol. 2001;155:1109-15.

8. Hirota T, Kunitoku N, Sasayama T, Marumoto T, Zhang DW, Nitta M, et al. Aurora-A and an interacting activator, the LIM protein Ajuba, are required for mitotic commitment in human cells. Cell. 2003;114:585-98.

9. Yoon Y, Cowley DO, Gallant J, Jones SN, Van Dyke T, Rivera-Perez JA. Conditional Aurora A deficiency differentially affects early mouse embryo patterning. Dev Biol. 2012;371:77-85.

10. Cowley DO, Rivera-Perez JA, Schliekelman M, He YJ, Oliver TG, Lu L, et al. Aurora-A Kinase Is Essential for Bipolar Spindle Formation and Early Development. Mol Cell Biol. 2009;29:1059-71.

11. Lu LY, Wood JL, Ye L, Minter-Dykhouse K, Saunders TL, Yu XC, et al. Aurora A Is Essential for Early Embryonic Development and Tumor Suppression. J Biol Chem. 2008:283:31785-90.

12. Tsai MY, Wiese C, Cao K, Martin O, Donovan P, Ruderman J, et al. A Ran signalling pathway mediated by the mitotic kinase Aurora A in spindle assembly. Nat Cell Biol. 2003;5:242-8.

13. Li Z, Rana TM. A kinase inhibitor screen identifies small-molecule enhancers of reprogramming and iPS cell generation. Nat Commun. 2012;3:1085

14. Lee DF, Su J, Ang YS, Carvajal-Vergara X, Mulero-Navarro S, Pereira CF, et al. Regulation of Embryonic and Induced Pluripotency by Aurora Kinase-p53 Signaling. Cell Stem Cell. 2012;11:179-94.

15. Sourisseau T, Maniotis D, McCarthy A, Tang C, Lord CJ, Ashworth A, et al. Aurora-A expressing tumour cells are deficient for homology-directed DNA double strand-break repair and sensitive to PARP inhibition. EMBO Mol Med. 2010;2:130-42.

16. Sankaran S, Crone DE, Palazzo RE, Parvin JD. Aurora-A kinase regulates breast cancer associated gene 1 inhibition of centrosome-dependent microtubule nucleation. Cancer Res. 2007;67:11186-94.

17. Yang G, Chang B, Yang F, Guo XQ, Cai KQ, Xiao X, et al. Aurora Kinase A Promotes Ovarian Tumorigenesis through Dysregulation of the Cell Cycle and Suppression of BRCA2. Clin Cancer Res. 2010;16:3171-81.

18. Do TV, Xiao F, Bickel LE, Klein-Szanto AJ, Pathak HB, Hua X, et al. Aurora kinase A mediates epithelial ovarian cancer cell migration and adhesion. Oncogene. 2013;33:539-549.

19. Dar AA, Belkhiri A, El-Rifai W. The aurora kinase A regulates GSK-3beta in gastric cancer cells. Oncogene. 2009;28:866-75.

20. Eyers PA, Erikson E, Chen LG, Maller JL. A novel mechanism for activation of the protein kinase aurora A. Curr Biol. 2003;13:691-7.

21. Ochi T, Fujiwara H, Suemori K, Azuma T, Yakushijin Y, Hato T, et al. Aurora-A kinase: a novel target of cellular immunotherapy for leukemia. Blood. 2009;113:66-74.

22. Park HS, Park WS, Bondaruk J, Tanaka N, Katayama H, Lee S, Spiess PE, Steinberg JR, Wang Z, Katz RL, et al: Quantitation of aurora kinase A gene copy number in urine sediments and bladder cancer detection. J Natl Cancer Inst 2008, 100:1401-1411.

23. Tanaka T, Kimura M, Matsunaga K, Fukada D, Mori H, Okano Y. Centrosomal kinase AIK1 is overexpressed in invasive ductal carcinoma of the breast. Cancer Res. 1999;59:2041-4. 
24. Watanabe T, Imoto I, Katahira T, Hirasawa A, Ishiwata I, Emi M, et al. Differentially regulated genes as putative targets of amplifications at $20 \mathrm{q}$ in ovarian cancers. Jpn J Cancer Res. 2002;93:1114-22.

25. Bischoff JR, Anderson L, Zhu YF, Mossie K, Ng L, Souza B, et al. A homologue of Drosophila aurora kinase is oncogenic and amplified in human colorectal cancers. EMBO J. 1998;17:3052-65.

26. Li DH, Zhu JJ, Firozi PF, Abbruzzese JL, Evans DB, Cleary K, et al. Overexpression of oncogenic STK15/BTAK/aurora A kinase in human pancreatic cancer. Clin Cancer Res. 2003;9:991-7.

27. Kamada K, Yamada Y, Hirao T, Fujimoto H, Takahama Y, Ueno M, et al. Amplification/overexpression of Aurora-A in human gastric carcinoma: Potential role in differentiated type gastric carcinogenesis. Oncol Rep. 2004;12:593-9.

28. Yang SB, Zhou XB, Zhu HX, Quan LP, Bai JF, He J, et al. Amplification and overexpression of Aurora-A in esophageal squamous cell carcinoma. Oncol Rep. 2007;17:1083-8.

29. Rojanala S, Han HY, Munoz RM, Browne W, Nagle R, Von Hoff DD, et al. The mitotic serine threonine kinase, Aurora-2, is a potential target for drug development in human pancreatic cancer. Mol Cancer Ther. 2004;3:451-7.

30. Jeng YM, Peng SY, Lin CY, Hsu HC. Overexpression and amplification of Aurora-A in hepatocellular carcinoma. Clin Cancer Res. 2004;10:2065-71.

31. Cammareri P, Scopelliti A, Todaro M, Eterno V, Francescangeli F, Moyer MP, et al. Aurora-a is essential for the tumorigenic capacity and chemoresistance of colorectal cancer stem cells. Cancer Res. 2010;70:4655-65.

32. Lee HH, Zhu YS, Govindasamy KM, Gopalan G. Downregulation of Aurora-A overrides estrogen-mediated growth and chemoresistance in breast cancer cells. Endocr Relat Cancer. 2008;15:765-75.

33. Wang $X$, Zhou YX, Qiao W, Tominaga $Y$, Ouchi M, Ouchi T, et al. Overexpression of aurora kinase $A$ in mouse mammary epithelium induces genetic instability preceding mammary tumor formation. Oncogene. 2006:25:7148-58

34. Zhou H, Kuang J, Zhong L, Kuo WL, Gray JW, Sahin A, et al. Tumour amplified kinase STK15/BTAK induces centrosome amplification, aneuploidy and transformation. Nat Genet. 1998;20:189-93.

35. Gorgun G, Calabrese E, Hideshima T, Ecsedy J, Perrone G, Mani M, et al. A novel Aurora-A kinase inhibitor MLN8237 induces cytotoxicity and cell-cycle arrest in multiple myeloma. Blood. 2010;115:5202-13.

36. Huck JJ, Zhang MK, McDonald A, Bowman D, Hoar KM, Stringer B, et al. MLN8054, an Inhibitor of Aurora A Kinase, Induces Senescence in Human Tumor Cells Both In vitro and In vivo. Mol Cancer Res. 2010;8:373-84.

37. Inoue M, Tsugane S. Epidemiology of gastric cancer in Japan. Postgrad Med J. 2005:81:419-24.

38. Devesa SS, Blot WJ, Fraumeni Jr JF. Changing patterns in the incidence of esophageal and gastric carcinoma in the United States. Cancer. 1998:83:2049-53.

39. Wiseman M. The second World Cancer Research Fund/American Institute for Cancer Research expert report. Food, nutrition, physical activity, and the prevention of cancer: a global perspective. Proc Nutr Soc. 2008;67:253-6.

40. Siegel R, Naishadham D, Jemal A. Cancer statistics, 2013. CA Cancer J Clin. 2013:63:11-30.

41. Turcotte S, Duranceau A. Gastroesophageal reflux and cancer. Thorac Surg Clin. 2005;15:341-52

42. Fujita Y, Sakakura C, Shimomura K, Nakanishi M, Yasuoka R, Aragane H, et al. Chromosome arm 20q gains and other genomic alterations in esophageal squamous cell carcinoma, as analyzed by comparative genomic hybridization and fluorescence in situ hybridization. Hepatogastroenterology. 2003:50:1857-63.

43. Sakakura C, Hagiwara A, Yasuoka R, Fujita Y, Nakanishi M, Masuda K, et al. Tumour-amplified kinase BTAK is amplified and overexpressed in gastric cancers with possible involvement in aneuploid formation. $\mathrm{Br} J$ Cancer. 2001;84:824-31.

44. Miao X, Sun T, Wang Y, Zhang X, Tan W, Lin D. Functional STK15 Phe31lle polymorphism is associated with the occurrence and advanced disease status of esophageal squamous cell carcinoma. Cancer Res. 2004;64:2680-3.

45. Ju H, Cho H, Kim YS, Kim WH, Ihm C, Noh SM, et al. Functional polymorphism $57 \mathrm{Val}>1 \mathrm{le}$ of aurora kinase $\mathrm{A}$ associated with increased risk of gastric cancer progression. Cancer Lett. 2006;242:273-9.

46. Tong $T$, Zhong $Y$, Kong J, Dong L, Song Y, Fu M, et al. Overexpression of Aurora-A contributes to malignant development of human esophageal squamous cell carcinoma. Clin Cancer Res. 2004;10:7304-10.
47. Dar AA, Zaika A, Piazuelo MB, Correa P, Koyama T, Belkhiri A, et al. Frequent overexpression of Aurora Kinase $A$ in upper gastrointestinal adenocarcinomas correlates with potent antiapoptotic functions. Cancer 2008;112:1688-98.

48. Sehdev V, Katsha A, Arras J, Peng D, Soutto M, Ecsedy J, et al. HDM2 regulation by AURKA promotes cell survival in gastric cancer. Clin Cancer Res. 2014;20:76-86.

49. Katsha A, Soutto M, Sehdev V, Peng D, Washington MK, Piazuelo MB, et al. Aurora kinase A promotes inflammation and tumorigenesis in mice and human gastric neoplasia. Gastroenterology. 2013;145:1312-22.

50. Tanaka E, Hashimoto Y, Ito T, Okumura T, Kan T, Watanabe G, et al. The clinical significance of Aurora-A/STK15/BTAK expression in human esophageal squamous cell carcinoma. Clin Cancer Res. 2005;11:1827-34.

51. Rugge M, Fassan M, Zaninotto G, Pizzi M, Giacomelli L, Battaglia G, et al. Aurora kinase A in Barrett's carcinogenesis. Hum Pathol. 2010;41:1380-6.

52. Dar AA, Belkhiri A, Ecsedy J, Zaika A, El-Rifai W. Aurora kinase A inhibition leads to p73-dependent apoptosis in p53-deficient cancer cells. Cancer Res. 2008:68:8998-9004.

53. Sehdev V, Katsha A, Ecsedy J, Zaika A, Belkhiri A, El-Rifai W. The combination of alisertib, an investigational Aurora kinase A inhibitor, and docetaxel promotes cell death and reduces tumor growth in preclinical cell models of upper gastrointestinal adenocarcinomas. Cancer. 2013;119:904-14.

54. Guan Z, Wang XR, Zhu XF, Huang XF, Xu J, Wang LH, et al. Aurora-A, a negative prognostic marker, increases migration and decreases radiosensitivity in cancer cells. Cancer Res. 2007;67:10436-44.

55. Katsha A, Arras J, Soutto M, Belkhiri A, El-Rifai W. AURKA regulates JAK2-STAT3 activity in human gastric and esophageal cancers. Mol Oncol. 2014. doi:10.1016/..molonc.2014.05.012.

56. Katayama H, Wang J, Treekitkarnmongkol W, Kawai H, Sasai K, Zhang H, et al. Aurora kinase-A inactivates DNA damage-induced apoptosis and spindle assembly checkpoint response functions of p73. Cancer Cell. 2012;21:196-211

57. Sehdev V, Peng D, Soutto M, Washington MK, Revetta F, Ecsedy J, et al. The aurora kinase A inhibitor MLN8237 enhances cisplatin-induced cell death in esophageal adenocarcinoma cells. Mol Cancer Ther. 2012;11:763-74.

58. Ewart-Toland A, Briassouli P, de Koning JP, Mao JH, Yuan J, Chan F, et al. Identification of Stk6/STK15 as a candidate low-penetrance tumorsusceptibility gene in mouse and human. Nat Genet. 2003;34:403-12.

59. Hienonen T, Salovaara R, Mecklin JP, Jarvinen H, Karhu A, Aaltonen LA Preferential amplification of AURKA 91A (lle31) in familial colorectal cancers. Int J Cancer. 2006:118:505-8.

60. Fu Z, Regan K, Zhang LZ, Muders MH, Thibodeau SN, French A, et al. Deficiencies in Chfr and Mlh1 synergistically enhance tumor susceptibility in mice. J Clin Invest. 2009;119:2714-24.

61. Belt EJ, Brosens RP, Delis-van Diemen PM, Bril H, Tijssen M, van Essen DF, et al. Cell cycle proteins predict recurrence in stage II and III colon cancer. Ann Surg Oncol. 2012;19 Suppl 3:S682-92.

62. Burum-Auensen E, De Angelis PM, Schjolberg AR, Kravik KL, Aure M, Clausen OP. Subcellular localization of the spindle proteins Aurora A, Mad2, and BUBR1 assessed by immunohistochemistry. J Histochem Cytochem. 2007;55:477-86

63. Tseng YS, Lee JC, Huang CYF, Liu HS. Aurora-A overexpression enhances cell-aggregation of Ha-ras transformants through the MEK/ERK signaling pathway. BMC Cancer. 2009;9:435-446.

64. Kuratnik A, Senapati VE, Verma R, Mellone BG, Vella AT, Giardina C. Acute sensitization of colon cancer cells to inflammatory cytokines by prophase arrest. Biochem Pharmacol. 2012;83:1217-28.

65. Shionome $Y$, Lin WH, Shiao HY, Hsieh HP, Hsu JT, Ouchi T. A novel aurora-A inhibitor, BPR1K0609S1, sensitizes colorectal tumor cells to 5-fluorofracil (5-FU) treatment. Int J Biol Sci. 2013;9:403-11.

66. Ratushny V, Pathak HB, Beeharry N, Tikhmyanova N, Xiao F, Li T, et al. Dual inhibition of SRC and Aurora kinases induces postmitotic attachment defects and cell death. Oncogene. 2012;31:1217-27.

67. Warner SL, Munoz RM, Bearss DJ, Grippo P, Han H, Von Hoff DD. Pdx-1-driven overexpression of aurora a kinase induces mild ductal dysplasia of pancreatic ducts near islets in transgenic mice. Pancreas. 2008;37:e39-44.

68. Zhu J, Abbruzzese JL, Izzo J, Hittelman WN, Li D. AURKA amplification, chromosome instability, and centrosome abnormality in human pancreatic carcinoma cells. Cancer Genet Cytogenet. 2005;159:10-7. 
69. Hata T, Furukawa T, Sunamura M, Egawa S, Motoi F, Ohmura N, et al. RNA interference targeting aurora kinase a suppresses tumor growth and enhances the taxane chemosensitivity in human pancreatic cancer cells. Cancer Res. 2005;65:2899-905.

70. Arbitrario JP, Belmont BJ, Evanchik MJ, Flanagan WM, Fucini RV, Hansen SK, et al. SNS-314, a pan-Aurora kinase inhibitor, shows potent anti-tumor activity and dosing flexibility in vivo. Cancer Chemother Pharmacol. 2010;65:707-17.

71. Lin Y, Richards FM, Krippendorff BF, Bramhall JL, Harrington JA, Bapiro TE, et al. Paclitaxel and CYC3, an aurora kinase A inhibitor, synergise in pancreatic cancer cells but not bone marrow precursor cells. Br J Cancer. 2012;107:1692-701.

72. Hamidi T, Cano CE, Grasso D, Garcia MN, Sandi MJ, Calvo EL, et al. Nupr1-aurora kinase A pathway provides protection against metabolic stress-mediated autophagic-associated cell death. Clin Cancer Res. 2012;18:5234-46.

73. Furukawa T, Kanai N, Shiwaku HO, Soga N, Uehara A, Horii A. AURKA is one of the downstream targets of MAPK1/ERK2 in pancreatic cancer. Oncogene. 2006;25:4831-9.

74. Lim KH, Brady DC, Kashatus DF, Ancrile BB, Der CJ, Cox AD, et al. Aurora-A Phosphorylates, Activates, and Relocalizes the Small GTPase RalA. Mol Cell Biol. 2010;30:508-23.

75. Neel NF, Stratford JK, Shinde V, Ecsedy JA, Martin TD, Der CJ, et al. Response to MLN8237 in Pancreatic Cancer Is Not Dependent on RalA Phosphorylation. Mol Cancer Ther. 2014;13:122-33.

76. Yu CTR, Hsu JM, Lee YCG, Tsou AP, Chou CK, Huang CYF. Phosphorylation and stabilization of HURP by aurora-A: Implication of HURP as a transforming target of aurora-A. Mol Cell Biol. 2005;25:5789-800.

77. Tsou AP, Yang CW, Huang CYF, Yu RCT, Lee YCG, Chang CW, et al. Identification of a novel cell cycle regulated gene, HURP, overexpressed in human hepatocellular carcinoma. Oncogene. 2003;22:298-307.

78. Klein A, Flugel D, Kietzmann T. Transcriptional regulation of serine/threonine kinase-15 (STK15) expression by hypoxia and HIF-1. Mol Biol Cell. 2008;19:3667-75.

79. Gao P, Wang R, Shen JJ, Lin F, Wang X, Dong K, et al. Hypoxia-inducible enhancer/alpha-fetoprotein promoter-driven RNA interference targeting STK15 suppresses proliferation and induces apoptosis in human hepatocellular carcinoma cells. Cancer Sci. 2008;99:2209-17.

80. Cui SY, Huang JY, Chen YT, Song HZ, Huang GC, De W, et al. The role of Aurora A in hypoxia-inducible factor 1alpha-promoting malignant phenotypes of hepatocelluar carcinoma. Cell Cycle. 2013;12:2849-66.

81. Benten D, Keller G, Quaas A, Schrader J, Gontarewicz A, Balabanov S, et al. Aurora kinase inhibitor PHA-739358 suppresses growth of hepatocellular carcinoma in vitro and in a xenograft mouse model. Neoplasia. 2009;11:934-44.

82. Zhang C, Wu XH, Zhang MF, Zhu LC, Zhao R, Xu DQ, et al. Small Molecule R1498 as a Well-Tolerated and Orally Active Kinase Inhibitor for Hepatocellular Carcinoma and Gastric Cancer Treatment via Targeting Angiogenesis and Mitosis Pathways. PLoS One. 2013, 8.

83. Kelly KR, Ecsedy J, Medina E, Mahalingam D, Padmanabhan S, Nawrocki ST, et al. The novel Aurora A kinase inhibitor MLN8237 is active in resistant chronic myeloid leukaemia and significantly increases the efficacy of nilotinib. J Cell Mol Med. 2011;15:2057-70.

84. Manfredi MG, Ecsedy JA, Meetze KA, Balani SK, Burenkova O, Chen W, et al. Antitumor activity of MLN8054, an orally active small-molecule inhibitor of Aurora A kinase. Proc Natl Acad Sci U S A. 2007;104:4106-11.

85. Manfredi MG, Ecsedy JA, Chakravarty A, Silverman L, Zhang M, Hoar KM, et al. Characterization of Alisertib (MLN8237), an Investigational Small-Molecule Inhibitor of Aurora A Kinase Using Novel In Vivo Pharmacodynamic Assays. Clin Cancer Res. 2011;17:7614-24.

86. Kollareddy M, Zheleva D, Dzubak P, Brahmkshatriya PS, Lepsik M, Hajduch M. Aurora kinase inhibitors: progress towards the clinic. Invest New Drugs. 2012;30:2411-32.

87. Cervantes A, Elez E, Roda D, Ecsedy J, Macarulla T, Venkatakrishnan K, et al. Phase I pharmacokinetic/pharmacodynamic study of MLN8237, an investigational, oral, selective aurora a kinase inhibitor, in patients with advanced solid tumors. Clin Cancer Res. 2012;18:4764-74.

88. Briassouli P, Chan F, Savage K, Reis-Filho JS, Linardopoulos S. Aurora-A regulation of nuclear factor-kappaB signaling by phosphorylation of IkappaBalpha. Cancer Res. 2007;67:1689-95.

89. Yang H, He L, Kruk P, Nicosia SV, Cheng JQ. Aurora-A induces cell survival and chemoresistance by activation of Akt through a p53-dependent manner in ovarian cancer cells. Int J Cancer. 2006;119:2304-12.
90. Moretti L, Niermann K, Schleicher S, Giacalone NJ, Varki V, Kim KW, et al. MLN8054, a small molecule inhibitor of aurora kinase a, sensitizes androgen-resistant prostate cancer to radiation. Int J Radiat Oncol Biol Phys. 2011;80:1189-97.

91. Maris JM, Morton CL, Gorlick R, Kolb EA, Lock R, Carol H, et al. Initial testing of the aurora kinase A inhibitor MLN8237 by the Pediatric Preclinical Testing Program (PPTP). Pediatr Blood Cancer. 2010;55:26-34.

92. Qi W, Cooke LS, Liu X, Rimsza L, Roe DJ, Manziolli A, et al. Aurora inhibitor MLN8237 in combination with docetaxel enhances apoptosis and anti-tumor activity in mantle cell lymphoma. Biochem Pharmacol. 2011;81:881-90.

93. Mahadevan D, Stejskal A, Cooke LS, Manziello A, Morales C, Persky DO, et al. Aurora A Inhibitor (MLN8237) plus Vincristine plus Rituximab Is Synthetic Lethal and a Potential Curative Therapy in Aggressive B-cell Non-Hodgkin Lymphoma. Clin Cancer Res. 2012;18:2210-9.

94. Dees EC, Cohen RB, von Mehren M, Stinchcombe TE, Liu H, Venkatakrishnan K, et al. Phase I study of aurora A kinase inhibitor MLN8237 in advanced solid tumors: safety, pharmacokinetics, pharmacodynamics, and bioavailability of two oral formulations. Clin Cancer Res. 2012;18:4775-84.

95. Melichar B, Adenis A, Lockhart AC, Bennouna J, Dees EC, Kayaleh O, et al. Safety and activity of alisertib, an investigational aurora kinase A inhibitor, in patients with breast cancer, small-cell lung cancer, non-small-cell lung cancer, head and neck squamous-cell carcinoma, and gastro-oesophageal adencarcinoma: a five-arm phase 2 study. Lancet Oncol. 2015;16:395-405.

96. Fletcher GC, Brokx RD, Denny TA, Hembrough TA, Plum SM, Fogler WE, et al. ENMD-2076 Is an Orally Active Kinase Inhibitor with Antiangiogenic and Antiproliferative Mechanisms of Action. Mol Cancer Ther. 2011;10:126-37.

97. Wang X, Sinn AL, Pollok K, Sandusky G, Zhang S, Chen L, et al. Preclinical activity of a novel multiple tyrosine kinase and aurora kinase inhibitor, ENMD-2076, against multiple myeloma. Br J Haematol. 2010;150:313-25.

98. Tentler JJ, Bradshaw-Pierce EL, Serkova NJ, Hasebroock KM, Pitts TM, Diamond JR, et al. Assessment of the in vivo antitumor effects of ENMD-2076, a novel multitargeted kinase inhibitor, against primary and cell line-derived human colorectal cancer xenograft models. Clin Cancer Res. 2010;16:2989-98.

99. Diamond JR, Eckhardt SG, Tan AC, Newton TP, Selby HM, Brunkow KL, et al. Predictive biomarkers of sensitivity to the aurora and angiogenic kinase inhibitor ENMD-2076 in preclinical breast cancer models. Clin Cancer Res. 2013;19:291-303.

100. Diamond JR, Bastos BR, Hansen RJ, Gustafson DL, Eckhardt SG, Kwak EL, et al. Phase I safety, pharmacokinetic, and pharmacodynamic study of ENMD-2076, a novel angiogenic and Aurora kinase inhibitor, in patients with advanced solid tumors. Clin Cancer Res. 2011;17:849-60.

101. Shimomura T, Hasako S, Nakatsuru Y, Mita T, Ichikawa K, Kodera T, et al. MK-5108, a Highly Selective Aurora-A Kinase Inhibitor, Shows Antitumor Activity Alone and in Combination with Docetaxel. Mol Cancer Ther. 2010;9:157-66.

102. Chefetz I, Holmberg JC, Alvero AB, Visintin I, Mor G. Inhibition of Aurora-A kinase induces cell cycle arrest in epithelial ovarian cancer stem cells by affecting NFkB pathway. Cell Cycle. 2011;10:2206-14.

103. Kretzner L, Scuto A, Dino PM, Kowolik CM, Wu J, Ventura $P$, et al. Combining histone deacetylase inhibitor vorinostat with aurora kinase inhibitors enhances lymphoma cell killing with repression of c-Myc, hTERT, and microRNA levels. Cancer Res. 2011;71:3912-20.

104. Minton SE, LoRusso P, Lockhart AC, Saif M, Krishnamurthi SS, Pickett-Gies CA, et al. A phase I study of MK-5108, an oral aurora A kinase inhibitor, in both monotherapy and in combination with docetaxel in patients with advanced solid tumors. J Clin Oncol. 2010;28(15_suppl (May 20 Supplement), 2010):e13026.

105. Hook KE, Garza SJ, Lira ME, Ching KA, Lee NV, Cao J, et al. An integrated genomic approach to identify predictive biomarkers of response to the aurora kinase inhibitor PF-03814735. Mol Cancer Ther. 2012;11:710-9.

106. Otto T, Horn S, Brockmann M, Eilers U, Schuttrumpf L, Popov N, et al. Stabilization of N-Myc is a critical function of Aurora A in human neuroblastoma. Cancer Cell. 2009;15:67-78.

107. Prajapati S, Tu Z, Yamamoto Y, Gaynor RB. IKKalpha regulates the mitotic phase of the cell cycle by modulating Aurora A phosphorylation. Cell Cycle. 2006;5:2371-80.

108. Mazzera L, Lombardi G, Abeltino M, Ricca M, Donofrio G, Giuliani N, et al. Aurora and IKK kinases cooperatively interact to protect multiple myeloma cells from Apo2L/TRAIL. Blood. 2013;122:2641-53. 
109. Ouchi M, Fujiuchi N, Sasai K, Katayama H, Minamishima YA, Ongusaha PP, et al. BRCA1 phosphorylation by Aurora-A in the regulation of $\mathrm{G} 2$ to $\mathrm{M}$ transition. J Biol Chem. 2004;279:19643-8.

110. Kelly KR, Shea TC, Goy A, Berdeja JG, Reeder CB, McDonagh KT, et al. Phase I study of MLN8237-investigational Aurora A kinase inhibitor-in relapsed/ refractory multiple myeloma, Non-Hodgkin lymphoma and chronic lymphocytic leukemia. Invest New Drugs. 2014;32:489-99.

Submit your next manuscript to BioMed Central and take full advantage of:

- Convenient online submission

- Thorough peer review

- No space constraints or color figure charges

- Immediate publication on acceptance

- Inclusion in PubMed, CAS, Scopus and Google Scholar

- Research which is freely available for redistribution 